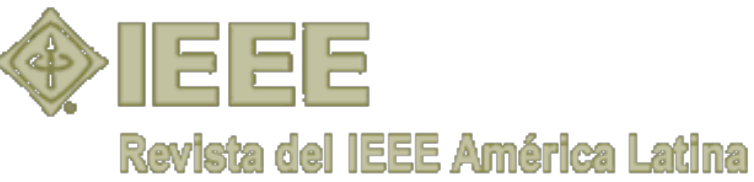

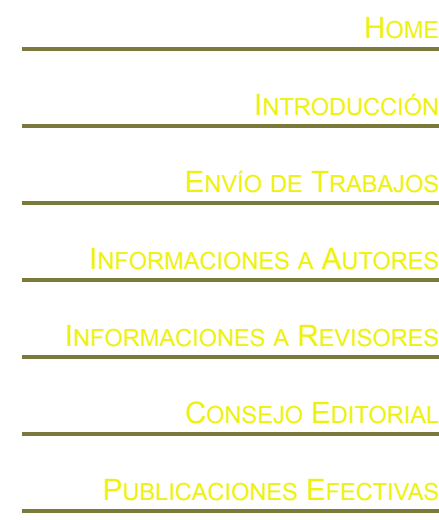

Patrocinador:

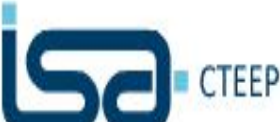

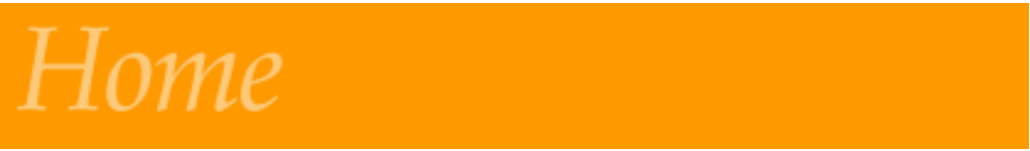

Bienvenidos a la Revista IEEE América Latina, una revista electrónica que está siendo publicada en español y portugués por la Región 9 del IEEE.

El lanzamiento del proyecto ha sido celebrado mundialmente tanto dentro como fuera del IEEE y se están presentando muchos trabajos. Esta revista no pretende competir con las publicaciones tradicionales en inglés del IEEE. Asume que hay trabajos de primer nivel en español y portugués que no están siendo publicados y que pueden compartirse a través de esta nueva revista.

Estamos interesados en publicar las mejores contribuciones, de modo que prestigiados ingenieros están colaborando en comités editoriales especializados. Han trabajado para seleccionar los trabajos que se ofrecen a través de la revista.

Agradecemos públicamente al Dr. Antonio Jardini y a su equipo por el gran trabajo voluntario que está asegurando el éxito de esta iniciativa.

Le invitamos a continuar enviando sus contribuciones. La Revista ha sido incluida en IEEEXplore http://ieeexplore.ieee.org/ ISSN: 1548-0992 


\title{
Modeling Workplace Evacuation Behaviors Using Intelligent Agents
}

\author{
M. A. Ramos, V. Muñoz, E. Castellanos, Member, IEEE and F.F. Ramos, Member, IEEE.
}

\begin{abstract}
A major challenge in artificial intelligence has been the development of autonomous agents (AAs) capable of displaying believable behaviors. To achieve such objective, the underlying architectures of these intelligent systems have been designed to incorporate Learning Classifier System that provides an adaptation naturally with the environment. It is expected that through the interaction of this type of components, AAs can implement more intelligent and believable behavior. Although the literature reports several computational models of behaviors, attention, and emotions developed to be included in cognitive agent architectures, these have been implemented as separated processes, disregarding essential interactions between these behaviors whose modeling and computational implementation may increase the believability of actions developed by AAs. In this paper, we propose an evolutive computational model. This model is designed to provide AAs with adequate mechanisms to attend and react to conditions and changes in the environment.
\end{abstract}

Keywords - Artificial Intelligence, Computational Modeling, LCS, Autonomous Agents.

\section{INTRODUCCIÓN}

$\mathrm{E}^{2}$ L ESTUDIO del comportamiento de los seres humanos es una necesidad de nuestros días debido al crecimiento de la población y a la concentración masiva de individuos en las ciudades. La necesidad de predecir y solucionar conflictos que pongan en peligro a los propios habitantes de las megalópolis, nos conlleva a estudiar el comportamiento de los individuos en diversas situaciones, que pueden ir desde un problema frecuente como es el tráfico, hasta pandemias masivas.

Las ciencias computacionales en lo general, y los campos de la inteligencia artificial, realidad virtual e interacción humano-computadora en lo particular, se enfrentan a un gran reto: la generación de agentes autónomos capaces de mimetizar el comportamiento de los seres humanos. Actualmente, se cuenta con arquitecturas cognitivas para crear agentes inteligentes, estas arquitecturas integran funciones de tipo afectivo y emocional. Pero también es indispensable considerar la percepción del ambiente y sus implicaciones: procesos de toma de decisiones, planificación y acciones. Estos últimos se consideran como aspectos independientes de la arquitectura debido a la complejidad y tiempo de respuesta del sistema.

M. A. Ramos, Universidad Autónoma del Estado de México, Estado de México, México, marco.corchado@gmail.com

V. Muñoz, Universidad Autónoma del Estado de México, Estado de México, México,vmunozj@uaemex.mx

E. Castellanos, Universidad Autónoma del Estado de México, Estado de México, México, hola@erickcastellanos.mx

F. F. Ramos, Cinvestav Unidad Guadalajara, Jalisco, México, framos@gdl.cinvestav.mx

Corresponding author: E. Castellanos.
Las áreas de conocimiento de neurociencias, neurocomputación y las propias del estudio del comportamiento humano, mencionan que el comportamiento es el resultado que engloba las diferentes funciones del cerebro, tales como: la atención, la emoción, la visión, las partes motoras del organismo, que en conjunto manifiestan un comportamiento único de cada individuo. En el mismo sentido, manifiestan que el proceso de adaptación al ambiente permite retroalimentar al individuo en cada situación para que este alcance sus metas y objetivos propuestos.

En este artículo se presenta un sistema clasificador de aprendizaje ( $L C S$, por sus siglas en inglés) para la generación de agentes autónomos que permita su adaptación a los cambios del medio ambiente en el que se encuentran inmersos. Los comportamientos no predefinidos deberán emerger por simple presión del ambiente y evolución de los mismos.

Adicionalmente, tomaremos los paradigmas de los Juegos Serios para recrear fenómenos reales en la vida cotidiana del ser humano con el objetivo principal de predecir y anticipar situaciones que pongan en riesgo la integridad de los individuos. Por ejemplo, existen países que sufren de movimientos de tierra (sismos) que generan graves daños a la población y desestabilizan a los gobiernos. Los daños por salud puede ser otro claro ejemplo que está directamente ligado al comportamiento del individuo.

En este artículo nos concentramos en un caso de estudio basado en la evacuación de un centro de trabajo ante la presencia de un fenómeno no controlado, en donde el comportamiento del individuo se ve afectado.

Para nuestro caso de estudio, que implica un centro de trabajo que denominamos ambiente, los agentes llevan o realizan actividades propias del centro, como es el puesto de trabajo físico en donde una entidad deberá realizar un comportamiento cotidiano. La evacuación deberá suceder al momento en que un evento no controlado dentro del ambiente se presente, por ejemplo, una alarma o solicitud de evacuación, donde las tareas o actividades de los agentes deberán ser interrumpidas inmediatamente para desalojar el espacio.

Los comportamientos de los agentes son clasificados de acuerdo a diferentes roles con la finalidad de llevar un control. Sin embargo, estos roles no limitan el comportamiento individual de cada uno de los agentes, debido a que estos usan el paradigma de creencias, deseos e intenciones (BDI, por sus siglas en inglés). Las creencias son todo aquello que se considera como conocimiento inicial del entorno y sus responsabilidades. Los deseos son los objetivos que debe cumplir el agente. Y las intenciones es el plan que el agente ejecutará para cumplir sus objetivos. 
Este artículo está organizado de la siguiente manera. En la sección II, presentamos la manera de generar agentes autónomos que muestren comportamientos realistas en un centro de trabajo atendiendo sus diferentes roles. En la sección III, se presenta el $L C S$ y sus principales características para la generación de comportamientos. En la Sección IV, se presenta el ambiente que corresponde al caso de estudio, en el cual se modeló el centro de trabajo. Finalmente en la sección V, presentamos los resultados obtenidos al utilizar los paradigmas de los juegos serios, que nos permitió realizar simulaciones para predecir y anticipar contingencias en eventos no controlados.

\section{AGENTES AUTÓNOMOS}

Un agente autónomo debe ser capaz de moverse dentro del ambiente sin ninguna supervisión, y capaz de tomar decisiones en función de la información proveniente del propio ambiente, de otros agentes y por supuesto del usuario ([1],[2],[3]). La necesidad del agente autónomo de contar con una conciencia propia crea la emergencia de comportamientos que son esenciales para las técnicas de predicción. Por lo tanto, un agente podrá ser una entidad física o virtual con las siguientes capacidades:

- Capaz de aprender e interactuar en un medio ambiente virtual.

- Capaz de comunicarse con otros agentes.

- Capaz de modificar sus creencias en función de los logros de sus objetivos individuales y de su satisfacción.

- Capaz de optimizar sus propios recursos.

- Capaz de percibir su ambiente para captar más recursos.

- Capaz de crear modelos abstractos del ambiente para la toma de decisiones.

- Capaz de transferir su conocimiento a otros agentes.

Por lo tanto, los agentes autónomos son herramientas que nos permiten resolver problemas complejos gracias al aprendizaje, adaptación y a su evolución casi al mismo tiempo que el medio ambiente. No obstante, resulta complicado poder crear un sistema completo, lo que hace que muchos laboratorios propongan posibles soluciones y arquitecturas para generar este tipo de sistemas ([4],[5]).

\section{SISTEMA CLASIFICADOR DE APRENDIZAJE}

El LCS es una máquina de aprendizaje [6] que utiliza el medio ambiente para converger sobre las soluciones óptimas de acuerdo a los objetivos definidos del sistema. Cuenta con mecanismos para retroalimentar al sistema y adaptarlo a las nuevas condiciones que se presenten, lo que hace que pueda completar tareas complejas. La Figura 1 representa la arquitectura básica de un LCS.

La arquitectura básica o general de un LCS está compuesto por una interfaz que le permite percibir el ambiente llamado "captores". Los captores codifican la información en mensajes binarios con una longitud $\mathrm{L}$ que se envía a la base de reglas almacenadas. Tiempo después, el módulo de comparación "selección de reglas" obtiene la regla más adecuada para enviar a los "efectores" y realizar una acción en el ambiente. La regla que realizó la acción es recompensada o penalizada, según sea el caso, a través de un proceso de evaluación de acción realizada y la consecuente distribución de créditos.

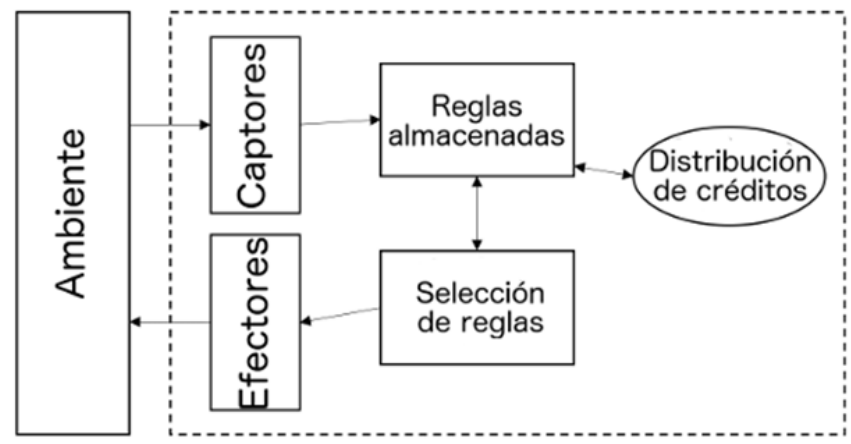

Figura 1. Arquitectura básica del LCS.

Múltiples mejoras se han realizado al LCS original. En este caso extenderemos el modelo XCS [7] debido a que este incorpora un mecanismo de aprendizaje (Q-learning) que permite la adaptación de las reglas, dejando atrás solo el criterio de recompensa y castigo. Además, el proceso de evolución se basa en la utilización de un algoritmo genético (AG), el cual se activa solamente cuando existen cambios importantes en el ambiente y no se cuentan con reglas que satisfagan las condiciones, lo que resulta en un sistema altamente reactivo. La Figura 2 muestra la arquitectura de un GXCS ([4],[6],[7]).

Es importante tener la libertad de poder representar cualquier tipo de información en un LCS permitiendo utilizar esta máquina en diferentes dominios y no restringirla a un área en específico. Por otra parte, sabemos que la mayoría de los ambientes son dinámicos lo que implica una codificación con mayor complejidad.

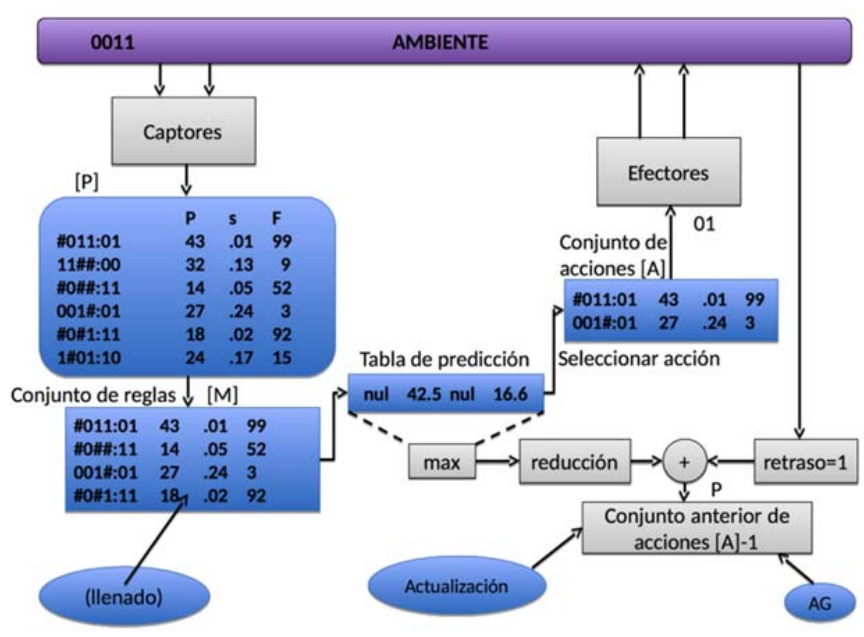

Figura 2. Arquitectura del GXCS. 


\section{AMBIENTE}

El ambiente representa los fenómenos físicos así como otro tipo de información que son pertinentes para reforzar el aprendizaje de los agentes autónomos. Los agentes habitan e interactúan con el medio ambiente, se adaptan y evolucionan en él [8]. Es el mismo ambiente que retroalimenta a los agentes mediante recompensas por las acciones realizadas y que aumentan el grado de satisfacción por el logro de los objetivos cumplidos.

Por otra parte, el ambiente hospeda al conjunto de agentes y conoce la población total de los mismos. Un punto importante del ambiente es considerar el aspecto temporal de la simulación. Contrariamente al mundo real, los mundos virtuales no trabajan en un tiempo continuo, lo que hace necesario controlar los estados del ambiente para evitar incoherencias. Es decir, en cada periodo de tiempo los agentes que habitan el ambiente deben actualizar su base de conocimiento de manera dinámica discriminando todos los estáticos (vegetación, muros, etc.). Por lo tanto, los agentes deben cumplir sus objetivos gracias a la presión que ejerce el medio ambiente en las creencias propias (aprendizaje).

La percepción es una parte importante de los agentes autónomos ya que estudia la información proveniente del medio ambiente. La principal dificultad se enfoca en tratar la información de manera correcta para permitir a los agentes la mejor adaptación. Para ello, se deben resolver dos premisas:

Premisa 1: Reconocer los mensajes pertinentes.

Premisa 2: Generar el mejor comportamiento coherente a la situación del medio ambiente, a los objetivos locales y globales.

La Figura 3 representa el plano arquitectónico de las oficinas ubicadas en el edificio $G$ de la Facultad de Ingeniería de la UAEM. Estas oficinas corresponden al espacio de trabajo de los profesores y estudiantes de posgrado. Este plano dibuja el centro de trabajo que se modelará y generará en 3D y que representará el ambiente en el caso de estudio.

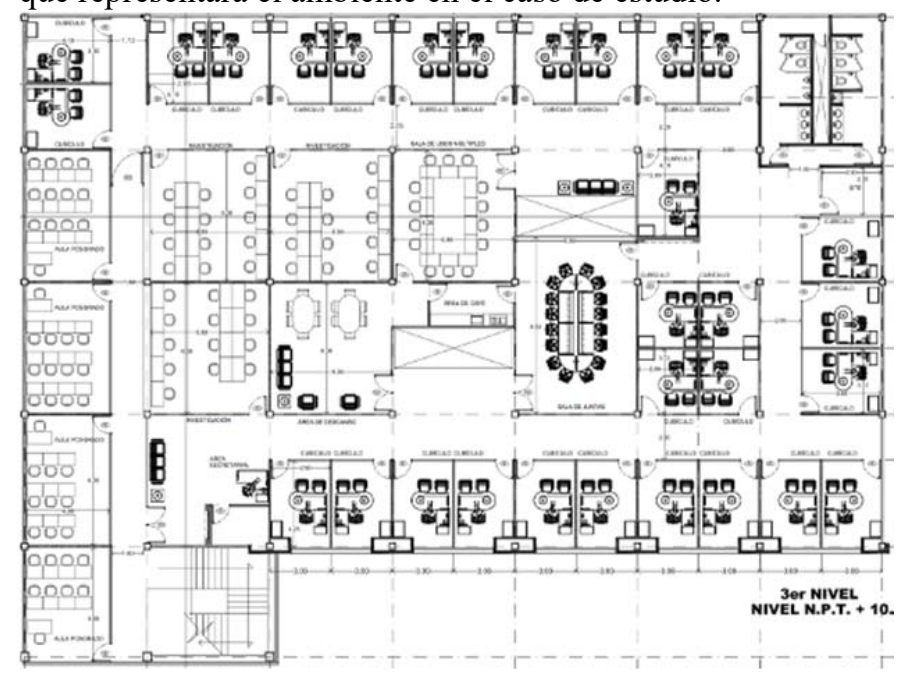

Figura 3. Plano arquitectónico (ambiente) del edificio G de la UAEM.
Los agentes deberán habitar este ambiente y realizar actividades cotidianas similares a los individuos que trabajan en este centro de trabajo ([1],[9]). En este sentido es que trabajaremos los diferentes roles, es decir, cada uno de estos roles definirá actividades típicas para cada uno de los agentes.

La información proveniente del medio ambiente es adquirida por los captores permitiendo al agente construir un modelo interno del ambiente (ver Figura 4), que le permitirá tomar la mejor acción. Paralelamente, se modifican los comportamientos individuales y colectivos, todo ello encaminado al logro de los objetivos.

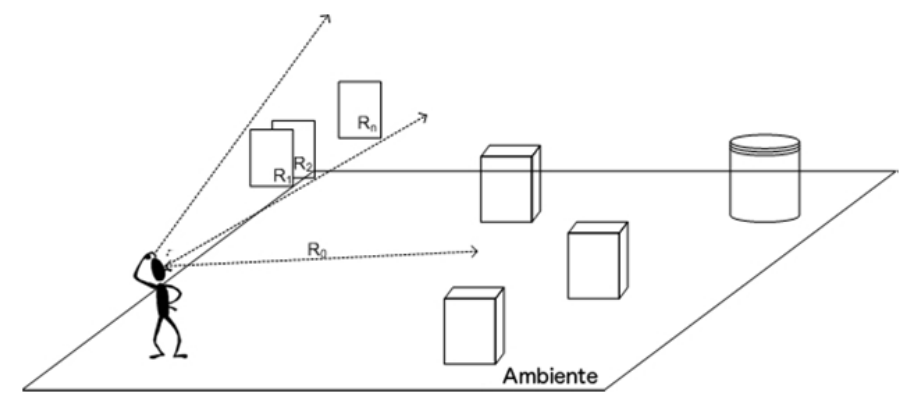

Figura 4. Modelo interno del ambiente que genera un agente.

Un modelo del medio ambiente se debe entender como una abstracción de la información que los captores proporcionan. Dotar a los agentes con la posibilidad de generar este tipo de modelos les permite evitar dificultades en acciones futuras y desencadenar una secuencia de acciones que genere un comportamiento más realista en el medio ambiente.

En las siguientes líneas describiremos la información que recibirán los captores y las acciones asociadas a las mismas, tal como se muestra en la Figura 5.

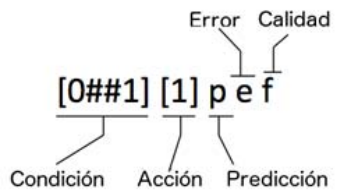

Figura 5. Codificación de los mensajes mediante los captores del sistema.

En la Figura 5 se muestran las diferentes partes que componen un mensaje que es tratado por el LCS, que dará respuesta a una situación dentro del ambiente.

La condición codifica directamente la percepción del agente en un tiempo $t$ en donde, para este caso en particular, 0 y 1 se deben cumplir. En esta codificación, el 0 indica que no debe estar presente la señal esperada por los captores, y el 1 indica la presencia de dicha señal. Por ejemplo, si la primer posición del mensaje, correspondiente a la sección de condición, estuviera asignada a la señal "pared al frente" y el mensaje indicara un 0 , esto implica que no se detecta ninguna pared al frente, y el 1 indicaría que sí se detecta una pared al frente. Además, un sistema LCS presenta la cualidad de poder filtrar la información no relevante al momento de realizar una acción, a lo cual llamamos "don't care", y es representado por un * o un \#. En el ejemplo mencionado, la presencia de un * indicaría que para esta regla en particular no nos interesa si 
existe, o no, una pared al frente. Aún más, al mensaje se le asocia una acción que deberá ser realizada mediante los efectores, además de una predicción y un error asociado a la predicción y finalmente una calidad de la regla [10].

La percepción la definimos como $\omega_{t}$, la cual corresponde a un estado del ambiente en el tiempo t. Para este modelo, $\omega_{s}$ es un vector de $p$ variables del medio ambiente.

$$
\omega_{s}=\left(e v_{s, 1}, e v_{s, 2}, \cdots, e v_{s, p}\right)
$$

donde cada $e v_{s, i}, 1 \leq i \leq p$ tiene valores de tipo real.

Una situación es un vector de estados del mundo definido por $S$.

$S=\left(S_{1}, S_{2}, \cdots, S_{l}\right)=\left(\omega_{s-l+1}, \omega_{s-l+2}, \cdots, \omega_{s-1}, \omega_{s}\right)$

Las acciones tomadas por los agentes modifican el estado del ambiente. En otras palabras, cada acción realizada por algún agente cambia una o más de las variables $e v_{s, i}$. Los cambios se producen entre $s$ y $s+1$ y pueden ser medidos por $r_{s}$, dado por:

$$
r_{s}=\omega_{s+1}-\omega_{s}
$$

Cada acción es una operación simple $\left(a_{1}^{s}, a_{2}^{s}, \cdots, a_{q}^{s}\right)$ que pueden ser ejecutadas por los efectores. Estas se definen a través de un vector que incluye las posibles acciones a realizar en el medio ambiente. Las acciones están ligadas directamente a las situaciones observadas en el ambiente, las cuales podrán ser ejecutadas de manera aleatoria. Por consiguiente, una acción es representada por un vector denominado $v^{a} \in N^{q}$, donde $q$ representa la longitud del vector de acciones a realizar en el ambiente.

Una de las principales ventajas de tener las acciones representada por un vector radica en la posibilidad de efectuarlas de manera aleatoria y continua. Esto produce comportamientos más realistas sin estar evaluando continuamente el estado del ambiente. Para controlar las acciones realizadas dentro del ambiente se cuenta con una ecuación de similaridad definida por:

$$
\operatorname{Sim}(a, b)=\exp \left(-\left\|v^{a}-v^{b}\right\|_{q}\right)
$$

donde $a$ y $b$ corresponden a las acciones a realizar de acuerdo a la percepción del ambiente. La similaridad producirá acciones continuas gracias a la función Sim que no necesita evaluar una nueva situación del ambiente.

Para nosotros la percepción será el conjunto de los estados del ambiente, las situaciones a evaluar y las acciones a realizar. Consecuentemente, cada una de las acciones realizadas se evaluará con base en el logro de objetivos a alcanzar por los agentes que habitan el medio ambiente. Estas tres condiciones deberán contar con la posibilidad de evolucionar de manera propia a los cambios directos e indirectos que sucedan en el ambiente.

Una de las principales dificultades que se tiene con los mensajes es el cómo construir las reglas iniciales, las cuales se irán adaptando con la evolución del sistema. Por esta razón decidimos utilizar el concepto de rol, el cual permite identificar a los diferentes tipos de agentes que habitarán nuestro centro de trabajo (ambiente). A manera de ejemplo presentaremos el rol de profesor, así como la lista de mensajes iniciales. En la Fig. 6 se muestra lo que percibe del ambiente y la acción a realizar.

Las reglas iniciales representan la base de conocimientos con la que cuenta el agente autónomo del ambiente que habita y quienes serán la base de aprendizaje inicial con el cual se irá adaptando al mismo. Conforme las reglas se vayan utilizando dentro del ambiente, estas serán evaluadas para determinar su eficiencia para el logro de los objetivos establecidos. A través de la evaluación se podrá determinar qué reglas se acercan a lo deseado y cuales no sirven para este propósito. Teniendo esta información cuantitativa se puede utilizar un mecanismo que permite la generación de nuevas reglas tomando como base las reglas con mejor evaluación e ir descartando las reglas con menor evaluación. Este mecanismo implica una evolución, una adaptación del conjunto de las reglas y es realizada gracias a un algoritmo genético incorporado directamente en la arquitectura del LCS. Después de algunas iteraciones dentro del ambiente, el conjunto de reglas convergerá hacia el resultado deseado a través del aprendizaje obtenido.

Cabe destacar que la propia arquitectura contempla el uso de reglas no aptas o no contempladas a priori a una situación pero que permiten al agente desenvolverse aun cuando la acción realizada acarree un mayor tiempo para alcanzar el éxito o el logro de objetivos (covering). Las acciones realizadas en el ambiente deben de respetar la coherencia en el mismo, por lo tanto, una acción no podrá ejecutarse si no respeta las reglas. Un ejemplo común es que un agente no podrá atravesar los muros o puertas.

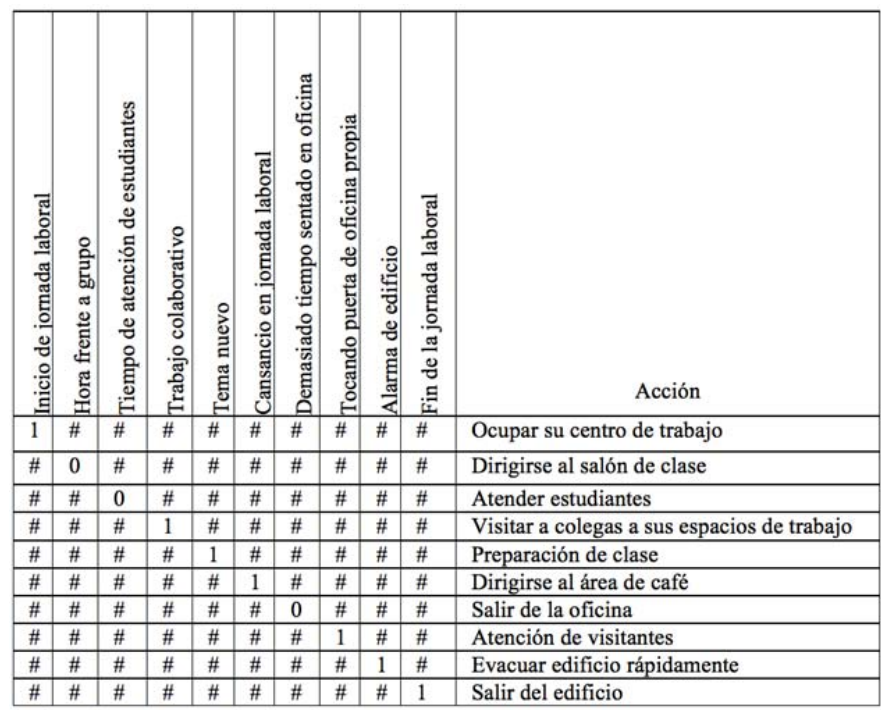

Figura 6. Reglas iniciales del LCS.

Otro factor importante a considerar en la concepción de las reglas es evitar que las reglas iniciales provoquen la subgeneralización de reglas, esto es, el uso excesivo del "don't care" quien provoca acciones no correctas dentro del ambiente. Esta condición implica la repetición de una misma acción para cualquier tipo de situación. Esto provocaría una falla global en el sistema, lo cual es una situación no deseada 
en cualquier ambiente.

\section{RESULTADOS}

Dentro del caso de estudio de esta propuesta, tenemos por el momento dos tipos de roles, el rol de profesor y el rol de estudiante. Estos roles son interpretados por los agentes. Los agentes deberán tener los rasgos humanos para hacer más creíble o realista la simulación ([11],[12]). Así mismo, el ambiente deberá contener la decoración actual del centro de trabajo.

La Fig. 7 y la Fig. 8 muestran los modelos 3D que serán utilizados para el caso de estudio.

Las propiedades físicas del ambiente serán tratadas directamente por el motor físico utilizado. Algunas de estas propiedades físicas son: la detección de colisiones, el efecto de la gravedad, el rendering y la iluminación.

Como se mencionó con anterioridad, se implementó el rol profesor y estudiante, y se definió la cantidad de actividades y acciones que pueden realizarse en el medio ambiente. Para lo cual se genera dentro del ambiente el mapa de las posiciones. Este mapa especifica los lugares donde los agentes pueden encontrase en un tiempo $t+1$. La Fig. 9 muestra el mapa por donde los agentes pueden desplazarse. La congruencia dentro del ambiente se basa en comportamientos de tipo "steering" ([13],[14]), de los cuales se implementan el de seguimiento y el de evasión. Los comportamientos como interacción, comunicación y colaboración son implementados directamente como reglas iniciales de la base de conocimiento de los agentes.

Los agentes se basan en una arquitectura tipo BDI que les permite modificar su base de conocimientos por las interacciones con el ambiente y con el LCS. Esto provoca que, no importando las actividades programadas y el logro de objetivos dentro del ambiente, estos puedan en todo momento interrumpirlas para asegurar su existencia para futuras acciones. Con ello logramos en nuestro caso de estudio que cuando exista una eventualidad no controlada, estos dejen de realizar las actividades desplazándose rápidamente a la salida del centro de trabajo que se está modelando.

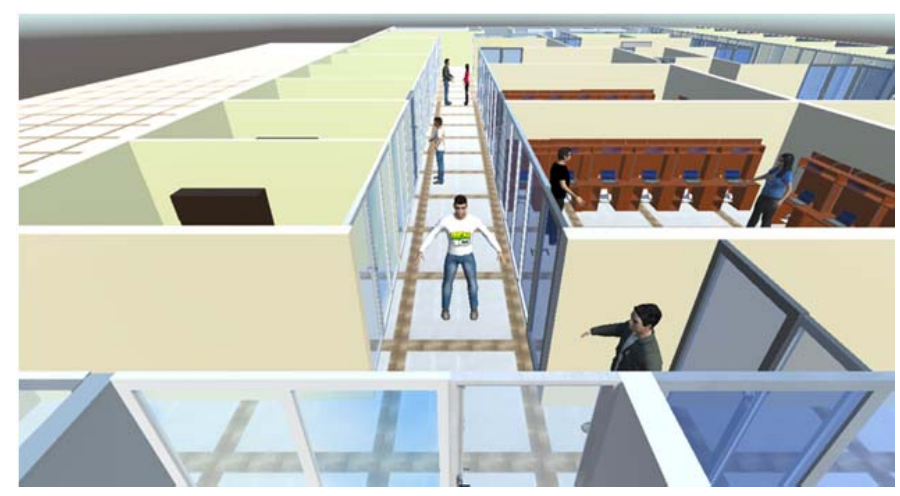

Figura 7. Modelo 3D de estudiantes que pertenecen al posgrado.

Comportamientos específicos como pánico, histeria, terror, etc., no están contemplados en este estudio, no obstante, son comportamientos que se consideran extender en el caso de estudio para la evacuación de centros de trabajo.

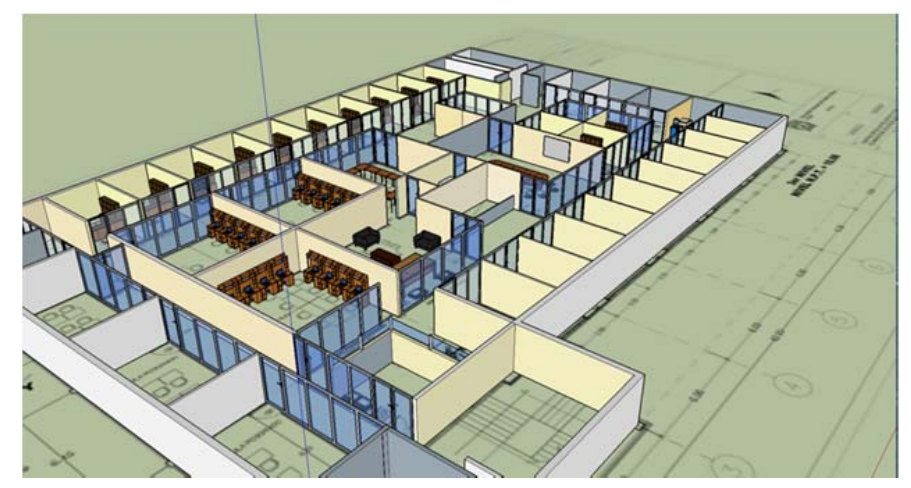

Figura 8. Modelo 3D del centro de trabajo correspondiente al edificio $\mathrm{G}$ de la UAEM.

Los resultados preliminares obtenidos de las simulaciones realizadas permiten observar emergencia de otros tipos de comportamientos, por ejemplo: empujar para lograr salir rápidamente de una puerta de dimensiones pequeñas. Este comportamiento emergente es sólo la muestra de un tipo de comportamiento más genérico que debe tomarse en cuenta: la interacción entre agentes y cómo pueden lograr de una mejor manera los objetivos globales del sistema a través de la transmisión de conocimiento adquirido y adaptado. Sin embargo, al ser esto un tema no trivial se ha dejado como parte del trabajo futuro a realizar. Este tipo de comportamientos pueden ser considerados para futuras evaluaciones y análisis de resultados.

Esto puede apoyar para el diseño arquitectónico de los centros de trabajo adecuando las instalaciones de acuerdo a los perfiles de los usuarios que laboran en este.

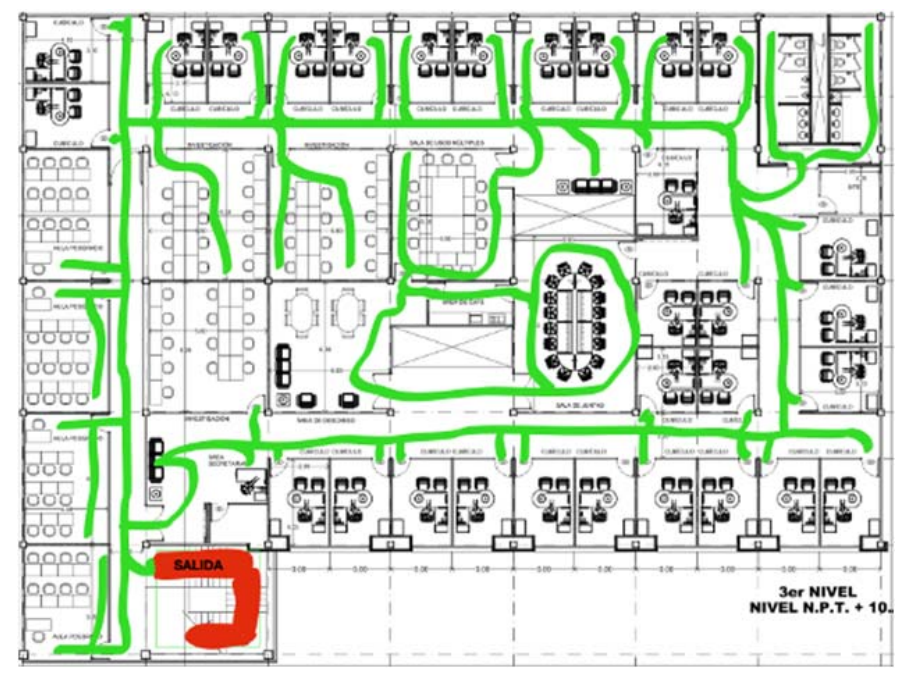

Figura 9. Mapa del medio ambiente por donde puede desplazarse el agente.

\section{CONCLUSIONES}

Los sistemas clasificadores LCS son una técnica de aprendizaje capaz de encontrar las soluciones dentro de 
ambientes complejos. La originalidad de este tipo de sistemas para explorar el ambiente es que permiten la aparición de nuevos comportamientos dentro del mismo. La modularidad en su implementación permite adicionar fácilmente otros componentes con la finalidad de mejorar las capacidades de los agentes.

La generación de comportamientos autónomos es un problema abierto y complejo que necesita de un mayor estudio, debido a la diversidad de comportamientos que manifiestan los seres humanos, considerando los diferentes perfiles sociales, psicológicos y neuronales.

El caso de estudio modelado y presentado en este artículo es una primera aproximación en donde se observan comportamientos realistas para la evacuación de un centro de trabajo tomando los paradigmas de los juegos serios.

Los comportamientos observados en este estudio no se presentan en otro tipo de simulaciones basadas en scripts, por lo cual obtuvimos resultados prometedores que permitirán crear nuevos comportamientos gracias al aprendizaje de los agentes autónomos en el medio ambiente que habitan.

Dichos resultados serán utilizados para extender el caso de estudio agregando la totalidad de roles desempeñados en el centro de trabajo.

Agradecemos las facilidades proporcionadas por la Universidad Autónoma del Estado de México (UAEM) y la intervención del Consejo Nacional de Ciencia y Tecnología (Conacyt).

\section{REFERENCIAS}

[1] C. Nikolai and G. Madey, "Tools of the Trade: A Survey of Various Agent Based Modeling Platforms," Journal of Artificial Societies and Social Simulation, vol. 12, no. 2, p. 2, 2009.

[2] Y. Demazeau, M. Pechoucek, J. M. C. Rodríguez, and J. B. Pérez, Advances on Practical Applications of Agents and Multiagent Systems: 9th International Conference on Practical Applications of Agents and Multiagent Systems, vol. 88. Springer Science \& Business Media, 2011.

[3] M. Wooldridge, An Introduction to MultiAgent Systems. Wiley, 2009.

[4] M. A. Ramos, A. Berro, and Y. Duthen, "Distributed Anticipatory System," in Advanced Distributed Systems: 5th International School and Symposium, ISSADS 2005, Guadalajara, Mexico, January 24-28, 2005, Revised Selected Papers, F. F. Ramos, V. Larios Rosillo, and H. Unger, Eds. Berlin, Heidelberg: Springer Berlin Heidelberg, 2005, pp. 443-451.

[5] J. P. Müller, The Design of Intelligent Agents: A Layered Approach. Springer, 1996.

[6] P. L. Lanzi, W. Stolzmann, and S. W. Wilson, Learning Classifier Systems: From Foundations to Applications. Springer Berlin Heidelberg, 2003.

[7] H. Olivier, S. Stéphane, B. Alain, and L. Hervé, "Generic classifiers systems and learning behaviours in virtual worlds," in Cyberworlds, 2004 International Conference on, 2004, pp. 113-120.

[8] D. Shiffman, "The Nature of Code: Simulating Natural Systems with Processing.: The Nature of Code," 2012.

[9] A. J. Stapleton, "Serious games: Serious opportunities," in Australian Game Developers" Conference, Academic Summit, Melbourne, 2004.

[10] J. Harland, D. N. Morley, J. Thangarajah, and N. Yorke-Smith, "An operational semantics for the goal life-cycle in BDI agents," Autonomous agents and multi-agent systems, vol. 28, no. 4, pp. 682719, 2014.

[11] D. R. Michael and S. L. Chen, Serious Games: Games That Educate, Train, and Inform. Muska \& Lipman/Premier-Trade, 2005.

[12] H. Kelly, K. Howell, E. Glinert, L. Holding, C. Swain, A. Burrowbridge, and M. Roper, "How to Build Serious Games," Commun. ACM, vol. 50, no. 7, pp. 44-49, Jul. 2007.

[13] C. J. Castle and A. T. Crooks, "Principles and concepts of agent-based modelling for developing geospatial simulations," 2006.
[14] C. W. Reynolds, "Steering behaviors for autonomous characters," in Game developers conference, 1999, vol. 1999, pp. 763-782.

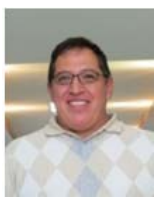

Marco A. Ramos Researcher professor in Artificial Intelligence and Virtual Reality in Universidad Autónoma del Estado de México. He got his PhD from Toulouse University on 2007, France. His research themes are: Artificial Life, animation techniques, distributed systems, Intelligent agents, etc.

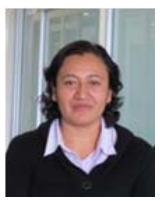

Vianney Muñoz Jiménez Researcher professor in image processing and Computational vision at Universidad Autónoma del Estado de México. In 2009, she received her $\mathrm{PhD}$ from Paris 13 University, France. Her research work is about computational vision, image processing, video compressing, etc.

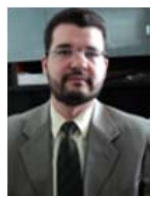

Erick Castellanos Invited Researcher at Universidad Autónoma del Estado de México. In 2015, he received his PhD from Cinvestav Unidad Guadalajara. Entrepreneur since 2004. His research interests includes: artificial life, virtual reality, intelligent systems, digital security, privacy and cryptography.

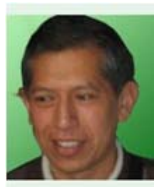

Félix F. Ramos He got his $\mathrm{PhD}$ from Compiègne Technologic University in France. He has experience working in education, research, technology development. In 1997 joined computer science department of Cinvestav as full time researcher. His research interests includes: Multi-agent Systems, Distributed Systems, animation of synthetic characters, virtual and augmented reality. 